\title{
Endoscopic ultrasound for differential diagnosis of malignant pancreatic cystic lesions. Case report and review of literature
}

\author{
Jacek Janiszewski 1, 2, Joanna Woźniak ${ }^{1,2}$, Iwona Kot-Gromuł', Maciej Michalak ${ }^{3,4}$, \\ Zygmunt Kozielec ${ }^{5,6}$, Dariusz Zadrożny7,8, Radosław Grabysa ${ }^{1,9}$ \\ 'Department of Internal Medicine and Gastroenterology, Warmian-Masurian Cancer Center \\ of the Ministry of the Interior and Administration's Hospital, Olsztyn, Poland \\ 2 Department of Cardiology and Internal Medicine, Faculty of Medical Sciences, \\ University of Warmia and Mazury, Olsztyn, Poland \\ ${ }^{3}$ Department of Radiology, Warmian-Masurian Cancer Center of the Ministry of the Interior \\ and Administration's Hospital, Olsztyn, Poland \\ ${ }^{4}$ Department of Radiology, Faculty of Medical Sciences, University of Warmia and Mazury, \\ Olsztyn, Poland \\ ${ }^{5}$ Department of Pathomorphology, Warmian-Masurian Cancer Center of the Ministry of \\ the Interior and Administration's Hospital, Olsztyn, Poland \\ ${ }^{6}$ Department of Pathomorphology, Faculty of Medical Sciences, University of Warmia and Mazury, \\ Olsztyn, Poland \\ ${ }^{7}$ Clinic of Surgical Oncology Warmian-Masurian Cancer Center of the Ministry of the Interior \\ and Administration's Hospital, Olsztyn, Poland \\ ${ }^{8}$ Department of Oncology, University of Warmia and Mazury, Olsztyn, Poland \\ ${ }^{9}$ Department of Pulmonology, Faculty of Medical Sciences, University of Warmia and Mazury, \\ Olsztyn, Poland
}

Correspondence:

Jacek Janiszewski Department of Internal Medicine and Gastroenterology, Warmian-Masurian Cancer Center of the Ministry of the Interior and Administration's Hospital 10-228 Olsztyn, Poland,

Wojska Polskiego 37 e-mail: jacekjaniszewski74@gmail.com phone: +48 691213621

Received:

5.10.2020

Accepted:

5.11.2020

DOI: 10.24292/01.OR.320051120 Copyright @ Medical Education. All rights reserved.

\section{ABSTRACT}

A 63-year-old female patient was admitted to the hospital for an in-depth diagnosis of accidentally found pancreatic cystic lesion. The lesion was detected by computed tomography scan and magnetic resonance imaging of the abdomen and identified as potentially malignant mucinous cystic neoplasm (MCN). Endoscopic ultrasound-guided fine-needle aspiration biopsy with the analysis of the fluid from the cyst was performed as well and it confirmed the malignancy of the cystic lesion. The patient was qualified for surgery and the final diagnosis based on histopathological examination of the surgical material confirmed MCN with the accompanying invasive pancreatic adenocarcinoma.

Key words: endoscopic ultrasound-guided fine-needle aspiration biopsy, pancreatic cancer, mucinous cystic neoplasm 


\section{INTRODUCTION}

An increasing number of abdominal imaging examinations resulted in higher cases of accidentally discovered cystic lesions in the pancreas. It is thought that about $40 \%$ of the cysts are neoplastic and therefore a fast and accurate diagnosis is crucial for further treatment of the patient [1]. The main goal of differential diagnosis is to determine, as closely as possible, the nature of the cyst to facilitate the selection of further treatment.

Differential diagnosis of malignant pancreatic cystic lesions (MPCL) needs to take into consideration whether the lesions are of malignant nature - intraductal papillary mucinous neoplasm (IPMN), serous cystadenocarcinoma (SCA), potentially malignantmucinous cystic neoplasm (MCN) or benign - serous cystic neoplasm (SCN) [2].

Endoscopic ultrasound (EUS), computed tomography (CT) and magnetic resonance imaging (MRI) are the currently available, modern methods for precise diagnosis of pancreatic cystic tumors. Endoscopic ultrasound-guided fine-needle aspiration biopsy (EUS-FNA) and the cytological examination of the fluid collected from the cyst (biochemical analysis, cytological analysis and mucus analysis) should be performed in order to obtain a comprehensive diagnosis of the lesions [3,4].

In this work we have presented the stages of differential diagnosis of the pancreatic cystic lesion found during preventive ultrasonography (USG) check-up of the abdomen. The application of EUS-FNA facilitated the formulation of the final diagnosis and the best course of treatment.

\section{CASE REPORT}

A 63-year-old female patient and a long-term tobacco smoker diagnosed during a preventative abdominal USG with a cystic lesion of the pancreatic tail was admitted to Internal Medicine and Gastroenterology ward for EUS-FNA of the lesion. The above change had already been visualised in CT and MRI scans of the abdomen. Multi-detector row computed tomography (MDCT) with thin-section scanning after iodine intravenous contrast administration was used as the imaging modality of choice for the initial detection and characterisation of the diagnosed pancreatic cystic lesion. Performed MDCT revealed oval, unilocular, macrocystic tumor with the diameter of $3 \mathrm{~cm}$, located in the body of pancreas. Typical imaging predictors of malignant transformation such as thick septations or peripheral enhancing nodules were not visible. The only area of concern was significant distal pancreatic atrophy with generalised narrowing of the main pan- creatic duct (MPD) (fig. 1). Subsequently, MRI of pancreas with magnetic resonance cholangiopancreatography (MRCP) was performed for an accurate depiction of the morphologic features of the lesion and with intent to demonstrate the relationship of the cyst to the pancreatic duct. The MRIT2-weighted images confirmed cystic nature of the lesion, without suspicious solid component and exclusion of internal debris, which is a typical finding for pancreatic pseudocyst (fig. 2).

FIGURE 1 .

Cystic pancreatic lesion with distal organ atrophy. Axial CT scan of the abdomen in venous phase after intravenous contrast administration.

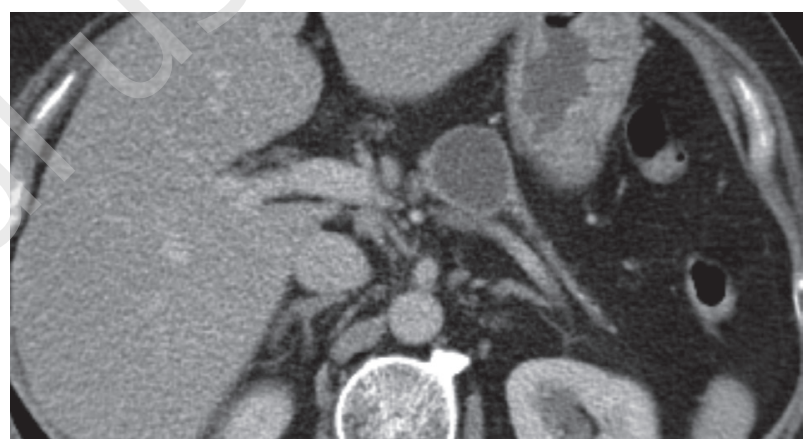

FIGURE 2.

A well-delineated, hyper intense pancreatic lesion with subtle irregularity of the cyst wall. Coronal Fat-Suppressed T2-weighted MRI scan.

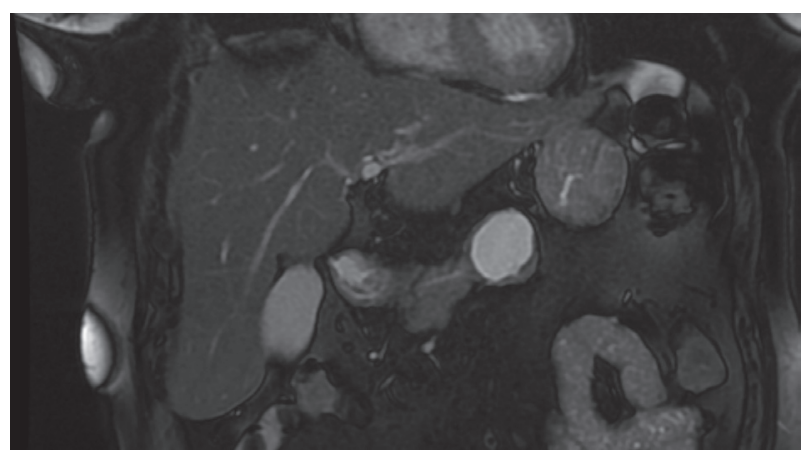

As a result of MRCP and the performed analysis of curved multiplanar reformatted (MPR) images from CT, communication between tumoral cyst with MPD was definitely excluded. Finally, a diagnosis of pancreatic MCN was made without evident radiological signs of malignancy.

EUS examination was performed during hospitalisation with Olympus GF-UCT 180 apparatus. The examination detected a cyst of $27.6 \times 32.8 \mathrm{~mm}$ in diameter in the tail of the pancreas, 
with a septum (fig. 3). At the base of the cyst septum, a thickening of the wall was detected with the presence of a mural nodule (fig. 4). The MPD, and the common bile duct (CBD) were not enlarged. The connection of the cyst with the pancreatic duct was not visualised. A biopsy of the cyst was performed with an Olympus EZ Shot 3 Plus $22 \mathrm{G}$ needle and 2 cubic $\mathrm{cm}$ of sticky liquid were obtained. The liquid was analysed for the concentration of carcinoembryonic antigen (CEA), amylase activity, glucose concentration and microscopic evaluation of the fluid after centrifugation (fig. 5).

\section{FIGURE 3.}

EUS image of a pancreatic cyst. The cyst diameter was determined to be $27.6 \times 32.8 \mathrm{~mm}$.

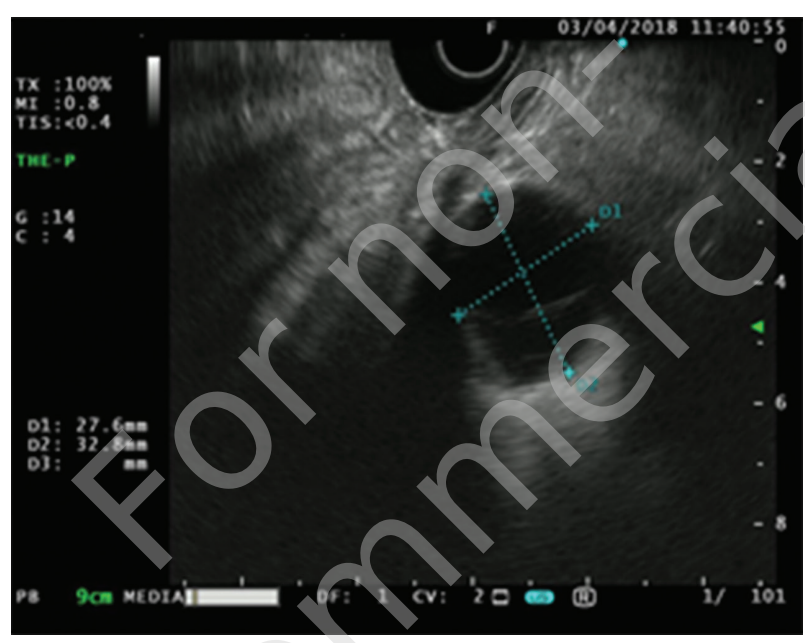

FIGURE 4.

EUS image of mural nodule protruding into the pancreatic cyst.

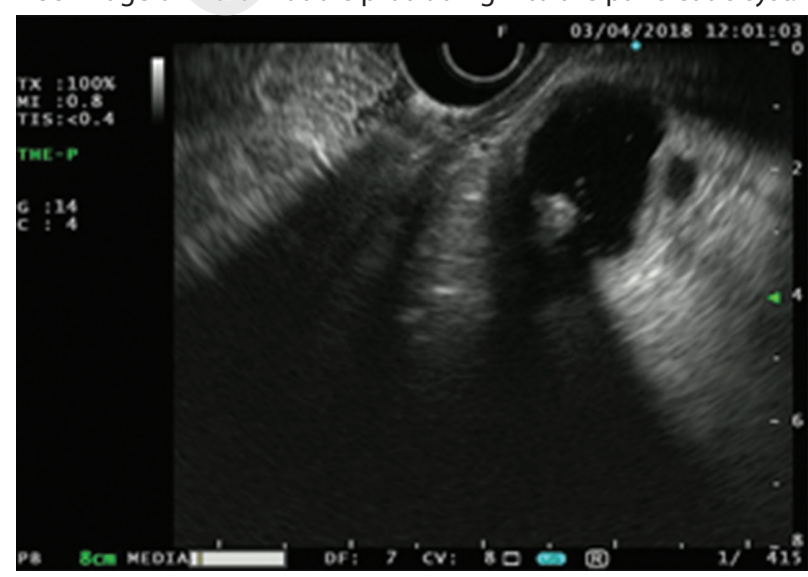

FIGURE 5.

EUS guided biopsy of a pancreatic cyst (Olympus EZ Shot 2 Plus 22 needle) to obtain fluid for biochemical and cytological evaluation.

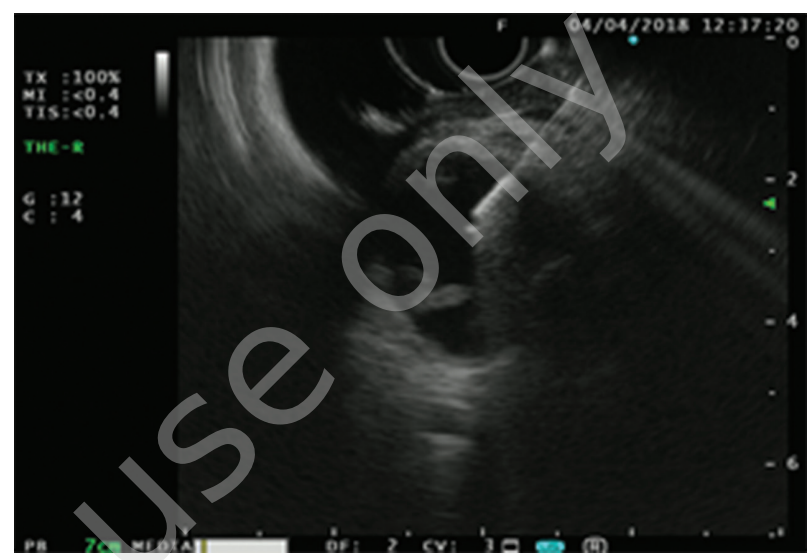

The obtained results showed high concentration of CEA (54254.00 ng/ml), low glucose concentration (7 mg/dl) and low amylase activity (14U/l). Erythrocytes were dominant in the microscopic smears of the fluid obtained after centrifugation, however, other morphotic blood elements were present as well. Single, leached cells, mostly naked nuclei of ambiguous nature were detected as well, however their presence did not warrant diagnosis. The obtained results indicated that the lesion was a mucous cyst [5-7].

Once the case analysis had been carried out, and having taken into consideration the ultrasound image of the lesion as well as the laboratory results, a diagnosis of MCN mucinous cystic neoplasm (MCN) was made. Due to risk factors of cyst malignancy, such as: the cyst diameter $\geq 3 \mathrm{~cm}$, the presence of the wall nodule, the thickening of the cyst wall, the presence of mucus with high concentration of CEA, and low glucose concentration, the patient was qualified for surgery [6-9].

The patient underwent peripheral pancreatic resection with splenectomy and lymphadenectomy. The procedure and the postoperative period were completed without complications.

Macroscopic analysis of the material carried out by a pathologist showed a fragment of the pancreas, $7.5 \times 5.5 \times 3 \mathrm{~cm}$ in size, with a cyst with a diameter of $3 \mathrm{~cm}$, filled with sticky, brown fluid. The lining of the cyst was smooth and shiny, with a nodule of $8 \mathrm{~mm}$ in diameter protruding into the lumen. Macroscopically analysed wall nodule tissue demonstrated high nuclear atypia and an adenocarcinoma with a diameter of $2 \mathrm{~mm}$ was detected within the nodule. 
Microscopic image ultimately confirmed $\mathrm{MCN}$ with an associated invasive, tubular - type adenocarcinoma of the pancreas (fig. 6).

FIGURE 6.

Pathological diagnosis of pancreatic adenocarcinoma within the mural nodule-mucinous cystic neoplasm (hematoxylin-eosin staining).

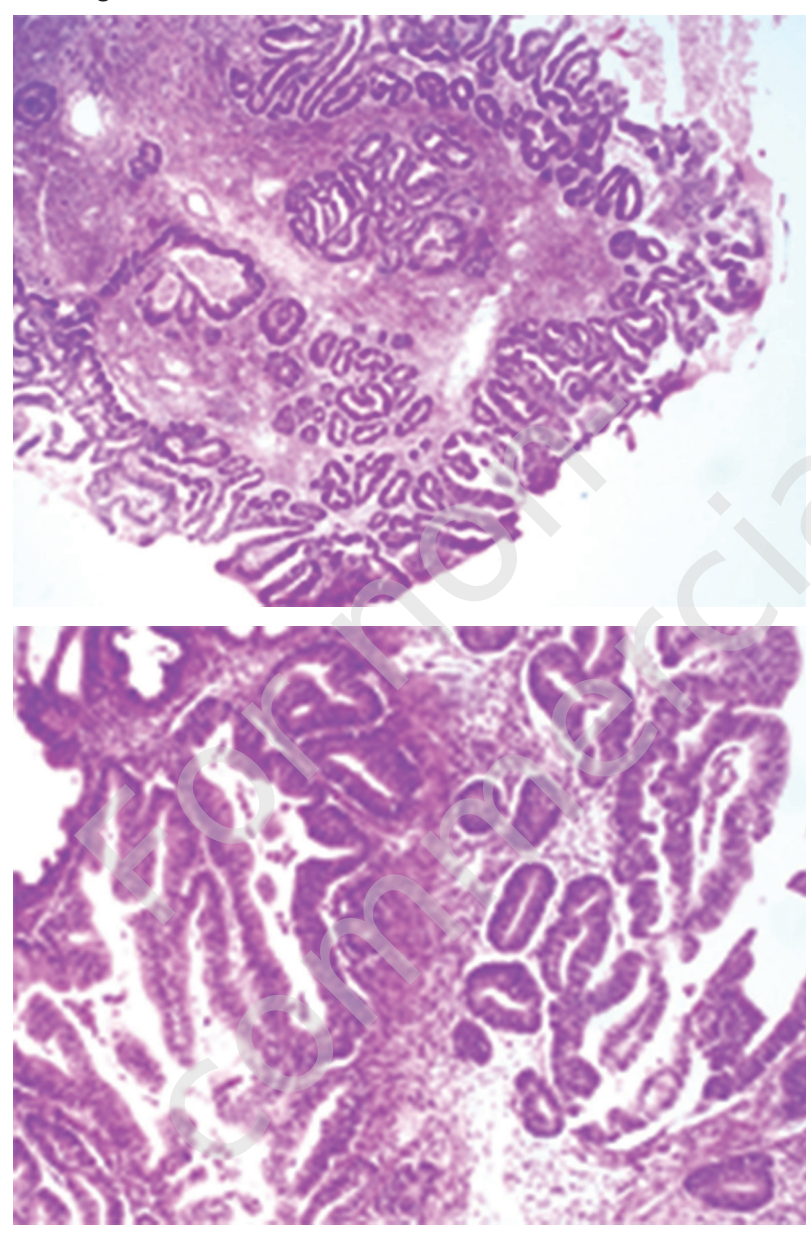

\section{DISCUSSION}

An increasing number of pancreatic cystic lesions are being detected due to the widespread use of abdominal imaging such as USG, CT and MRI. According to available data, pancreatic cysts have been detected in $2.5-13.5 \%$ of the patients who have been examined for reasons other than pancreatic diseases [10-12].

The ability to differentiate between benign pancreatic lesions, or with low malignant potential, such as pseudo-cysts or $\mathrm{SCN}$, and lesions with a high risk of turning malignant, e.g. IPMN or MCN has become a real challenge.

In the case described, EUS-FNA technique confirmed the size of the cyst, detected the thickening of the cyst wall and the presence of mural nodule.

The sample material obtained during the examination was analysed and the results of the analysis combined with EUS images facilitated making a decision to carry out the resection of the lesion [8].

EUS image correlated fully with the macroscopic image of the cyst in the post-procedure sample and the mural nodule described in EUS examination was confirmed in microscopic analysis as a mucous tumor with an invasive carcinoma $2 \mathrm{~mm}$ in diameter.

\section{SUMMARY}

The above-described case confirms the importance of endoscopic ultrasound-guided fine-needle aspiration biopsy technique in diagnosing the degree of malignancy in pancreatic cysts. Moreover, it clearly demonstrates that endoscopic ultrasound is a far more precise diagnostic tool than CT and MRI in imaging of minuscule changes inside the cyst, e.g. wall nodules. Our observation is consistent with the International Consensus Guidelines (ICG), which is currently the main factor qualifying the patient for surgery [8].

\section{References}

1. Hawes RH, Fockenes P. Endosonography. Saunders Elsevier. 2006.

2. Khalid A, Brugge W. ACG practice guidelines for the diagnosis and management of neoplastic pancreatic cysts. Am I Gastroenterol. 2007; 102(10): 2339-49.

3. Linder JD, Geenen JE, Catalano MF. Cyst fluid analysis obtained by EUS-guided FNA in the evaluation of discrete cystic neoplasms of the pancreas: a prospective single-center experience. Gastrointest Endosc. 2006; 64(5): 697-702.

4. Emerson RE, Randolph, Cramer HM. Endoscopic ultrasound-guided fine-needle aspiration cytology diagnosis of intraductal papillary mucinous neoplasm of the pancreas is highly predictive of pancreatic neoplasia. Diagn Cytopathol. 2006; 34(7): 457-62.

5. Al-Rashdan A, Schmidt CM, Al-Haddad M et al. Fluid analysis prior to surgical resection of suspected mucinous pancreatic cysts. A single centre experience. J Gastrointest Oncol. 2011; 2(4): 208-14. 
6. Bishop Pitman M, Lewandrowski K, Shen J et al. Pancreatic cysts: preoperative diagnosis and clinical management. Cancer Cytopathol. 2010; 118(1): 1-13. https://doi.org/10.1002/cncy.20059.

7. Levy P, Rebours V. The Role of Endoscopic Ultrasound in the Diagnosis of Cystic Lesions of the Pancreas. Visc Med. 2018; 34(3): 192-6. https://doi. org/10.1159/000489242.

8. Farrell JJ. Pancreatic cysts and guidelines. Dig Dis Sci. 2017; 62: 1827-39.

9. Bishop Pitman M. Revised international consensus guidelines for the management of patients with mucinous cysts. Cancer Cytopathol. 2012; 120(6): 361-5. https://doi.org/10.1002/cncy.21235.

10. Laffan TA, Horton KM, Klein AP et al. Prevalence of unsuspected pancreatic cysts on MDCT. Am J Roentgenol. $2008 ; 191: 802-7$.

11. de Jong K, Nio CY, Hermans JJ et al. High prevalence of pancreatic cysts detected by screening magnetic resonance imaging examinations. Clin Gastroenterol Hepatol. 2010; 8: 806-11.

12. Lee KS, Sekhar A, Rofsky NM et al. Prevalence of Incidental Pancreatic Cysts in the Adult Population on MR Imaging. Am J Gastroenterol. 2010; 105(9): 2079-84. 